\title{
Energy-efficient Deployment of Mobile Sensor Networks by PSO
}

\author{
Wu Xiaoling, Shu Lei, Wang Jin, Jinsung Cho' ${ }^{1}$, and Sungyoung Lee \\ Department of Computer Engineering, Kyung Hee University, Korea \\ \{xiaoling, sl8132, wangjin, sylee\}@oslab.khu.ac.kr \\ chojs@khu.ac.kr
}

\begin{abstract}
Sensor deployment is an important issue in designing sensor networks. In this paper, particle swarm optimization (PSO) approach is applied to maximize the coverage based on a probabilistic sensor model in mobile sensor networks and to reduce cost by finding the optimal positions for the clusterhead nodes based on a well-known energy model. During the coverage optimization process, sensors move to form a uniformly distributed topology according to the execution of algorithm at base station. The simulation results show that PSO algorithm has faster convergence rate than genetic algorithm based method while achieving the goal of energy efficient sensor deployment.
\end{abstract}

\section{Introduction}

Mobile sensor networks consist of sensor nodes that are deployed in a large area, collecting important information from the sensor field. Communication between the nodes is wireless. Since the nodes have very limited energy resources, the energy consuming operations such as data collection, transmission and reception must be kept at a minimum.

In most cases, a large number of wireless sensor devices can be deployed in hostile areas without human involved, e.g. by air-dropping from an aircraft for remote monitoring and surveillance purposes. Once the sensors are deployed on the ground, their data are transmitted back to the base station to provide the necessary situational information.

The deployment of mobile sensor nodes in the region of interest (ROI) where interesting events might happen and the corresponding detection mechanism is required is one of the key issues in this area. Before a sensor can provide useful data to the system, it must be deployed in a location that is contextually appropriate. Optimum placement of sensors results in the maximum possible utilization of the available sensors. The proper choice for sensor locations based on application requirements is difficult. The deployment of a static network is often either human monitored or random. Though many scenarios adopt random deployment for practical reasons such as

1 Prof. Jinsung Cho is the corresponding author. 
deployment cost and time, random deployment may not provide a uniform sensor distribution over the ROI, which is considered to be a desirable distribution in mobile sensor networks. Uneven node topology may lead to a short system lifetime.

The limited energy storage and memory of the deployed sensors prevent them from relaying data directly to the base station. It is therefore necessary to form a cluster based topology, and the cluster heads (CHs) provide the transmission relay to base station such as a satellite. And the aircraft carrying the sensors has a limited payload, so it is impossible to randomly drop thousands of sensors over the ROI, hoping the communication connectivity would arise by chance; thus, the mission must be performed with a fixed maximum number of sensors. In addition, the airdrop deployment may introduce uncertainty in the final sensor positions. These limitations motivate the establishment of a planning system that optimizes the sensor reorganization process after initial random airdrop deployment, which results in the maximum possible utilization of the available sensors.

There are lots of research work [1], [2], [3], [4], [12] related to the sensor nodes placement in network topology design. Most of them focused on optimizing the location of the sensors in order to maximize their collective coverage. However only a single objective was considered in most of the research papers, other considerations such as energy consumption minimization are also of vital practical importance in the choice of the network deployment. Self-deployment methods using mobile nodes [4], [9] have been proposed to enhance network coverage and to extend the system lifetime via configuration of uniformly distributed node topologies from random node distributions. In [4], the authors present the virtual force algorithm (VFA) as a new approach for sensor deployment to improve the sensor field coverage after an initial random placement of sensor nodes. The cluster head executes the VFA algorithm to find new locations for sensors to enhance the overall coverage. They also considered unavoidable uncertainty existing in the precomputed sensor node locations. This uncertainty-aware deployment algorithm provides high coverage with a minimum number of sensor nodes. However they assumed that global information regarding other nodes is available. In [1], the authors examined the optimization of wireless sensor network layouts using a multi-objective genetic algorithm (GA) in which two competing objectives are considered, total sensor coverage and the lifetime of the network. However the computation of this method is not inexpensive.

In this paper, we attempt to solve the coverage problem while considering energy efficiency using particle swarm optimization (PSO) algorithm, which can lead to computational faster convergence than genetic algorithm used to solve the deployment optimization problem in [1]. During the coverage optimization process, sensor nodes move to form a uniformly distributed topology according to the execution of algorithm at the base station. To the best of our knowledge, this is the first paper to solve deployment optimization problem by PSO algorithm.

In the next section, the PSO algorithm is introduced and compared with GA. Modeling of sensor network and the deployment algorithm is presented in section 3, followed by simulation results in section 4 . Some concluding remarks and future work are provided in section 5 . 


\section{Particle Swarm Optimization}

PSO, originally proposed by Eberhart and Kennedy [5] in 1995, and inspired by social behavior of bird flocking, has come to be widely used as a problem solving method in engineering and computer science.

The individuals, called, particles, are flown through the multidimensional search space with each particle representing a possible solution to the multidimensional problem. All of particles have fitness values, which are evaluated by the fitness function to be optimized, and have velocities, which direct the flying of the particles. PSO is initialized with a group of random solutions and then searches for optima by updating generations. In every iteration, each particle is updated by following two "best" factors. The first one, called pbest, is the best fitness it has achieved so far and it is also stored in memory. Another "best" value obtained so far by any particle in the population, is a global best and called gbest. When a particle takes part of the population as its topological neighbors, the best value is a local best and is called lbest. After each iteration, the pbest and gbest (or lbest) are updated if a more dominating solution is found by the particle and population, respectively.

The PSO formulae define each particle in the D-dimensional space as $X_{\mathrm{i}}=\left(x_{\mathrm{i} 1}, x_{i 2}\right.$, $\left.x_{i 3}, \ldots \ldots, x_{i D}\right)$ where $i$ represents the particle number, and $d$ is the dimension. The memory of the previous best position is represented as $P_{i}=\left(p_{i l}, p_{i 2}, p_{i 3} \ldots \ldots p_{i D}\right)$, and a velocity along each dimension as $V_{i}=\left(v_{i l}, v_{i 2}, v_{i 3} \ldots \ldots v_{i D}\right)$. The updating equation [6] is as follows,

$$
\begin{gathered}
v_{i d}=\varpi \times v_{i d}+c_{1} \times \operatorname{rand}() \times\left(p_{i d}-x_{i d}\right)+c_{2} \times \operatorname{rand}() \times\left(p_{g d}-x_{i d}\right) \\
x_{i d}=x_{i d}+v_{i d}
\end{gathered}
$$

where $\varpi$ is the inertia weight, and $c_{1}$ and $c_{2}$ are acceleration coefficients.

The role of the inertia weight $\varpi$ is considered to be crucial for the PSO' s convergence. The inertia weight is employed to control the impact of the previous history of velocities on the current velocity of each particle. Thus, the parameter $\varpi$ regulates the trade-off between global and local exploration ability of the swarm. A large inertia weight facilitates global exploration, while a small one tends to facilitate local exploration, i.e. fine-tuning the current search area. A suitable value for the inertia weight $\varpi$ balances the global and local exploration ability and, consequently, reduces the number of iterations required to locate the optimum solution. Generally, it is better to initially set the inertia to a large value, in order to make better global exploration of the search space, and gradually decrease it to get more refined solutions. Thus, a time-decreasing inertia weight value is used [7].

PSO shares many similarities with GA. Both algorithms start with a group of a randomly generated population, have fitness values to evaluate the population, update the population and search for the optimum with random techniques. However, PSO does not have genetic operators like crossover and mutation. Particles update themselves with the internal velocity. They also have memory, which is important to the algorithm [8]. 
Compared with GA, PSO is easy to implement, has few parameters to adjust, and requires only primitive mathematical operators, computationally inexpensive in terms of both memory requirements and speed while comprehensible. It usually results in faster convergence rates than GA. This feature suggests that PSO is a potential algorithm to optimize deployment in a sensor network.

\section{The Proposed Algorithm}

First of all, we present the model of mobile sensor network. We assume that each node knows its position in the problem space, all sensor members in a cluster are homogeneous and cluster heads are more powerful than sensor members. Communication coverage of each node is assumed to have a circular shape without any irregularity. The design variables are 2D coordinates of the sensor nodes, $\left\{\left(x_{1}, y_{1}\right),\left(x_{2}\right.\right.$, $\left.\left.y_{2}\right), \ldots ..\right\}$. Sensor nodes are assumed to have certain mobility. Many research efforts into the sensor deployment problem in mobile sensor networks [4, 9] make this sensor mobility assumption reasonable.

\subsection{Optimization of Coverage}

We consider coverage as the first optimization objective. It is one of the measurement criteria of QOS of a sensor network.

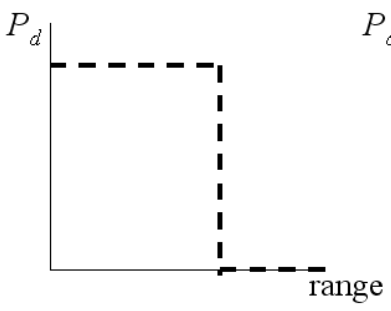

(a)

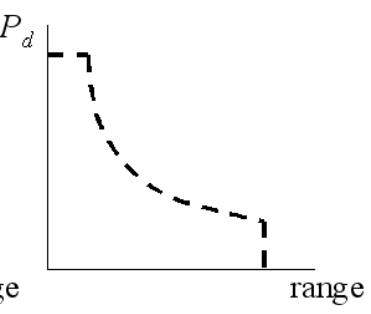

(b)

Fig. 1. Sensor coverage models (a) Binary sensor and (b) probabilistic sensor models

The coverage of each sensor can be defined either by a binary sensor model or a probabilistic sensor model as shown in Fig. 1. In the binary sensor model, the detection probability of the event of interest is 1 within the sensing range, otherwise, the probability is 0 . Although the binary sensor model is simpler, it is not realistic as it assumes that sensor readings have no associated uncertainty. In reality, sensor detections are imprecise, hence the coverage needs to be expressed in probabilistic terms. In many cases, cheap sensors such as omnidirectional acoustic sensors or ultrasonic sensor are used. Some practical examples [4] include AWAIRS at UCLA/RSC Smart Dust at UC Berkeley, the USC-ISI network, the DARPA SensIT systems/networks, the ARL Advanced Sensor Program systems/networks, and the DARPA Emergent Surveillance Plexus (ESP). For omnidirectional acoustic sensors or ultrasonic sensors, a longer distance between the sensor and the target generally implies a greater loss in 
the signal strength or a lower signal-to-noise ratio. This suggests that we can build an abstract sensor model to express the uncertainty in sensor responses. In other words, a sensor node that is closer to a target is expected to have a higher detection probability about the target existence than the sensor node that is further away from the target.

In this paper, the probabilistic sensor model given in Eq (3) is used, which is motivated in part by [11].

$$
c_{i j}(x, y)=\left\{\begin{array}{llc}
0, & \text { if } & r+r_{e} \leq d_{i j}(x, y) \\
e^{-\lambda a^{\beta}}, & \text { if } & r-r_{e}<d_{i j}(x, y)<r+r_{e} \\
1, & \text { if } & r+r_{e} \geq d_{i j}(x, y) .
\end{array}\right.
$$

The sensor field is represented by an $m \times n$ grid. An individual sensor node $s$ on the sensor field is located at grid point $(x, y)$. Each sensor node has a detection range of $r$. For any grid point $P$ at $(i, j)$, we denote the Euclidean distance between $s$ at $(x, y)$ and $P$ at $(i, j)$ as $d_{i j}(x, y)$, i.e., $d_{i j}(x, y)=\sqrt{(x-i)^{2}+(y-j)^{2}}$. Eq (3) expresses the coverage $c_{i j}(x, y)$ of a grid point at $(i, j)$ by sensor $s$ at $(x, y)$, in which $r_{e}\left(r_{e}<r\right)$ is a measure of the uncertainty in sensor detection, $a=d_{i j}(x, y)-\left(r-r_{e}\right)$, and $\lambda$ and $\beta$ are parameters that measure detection probability when a target is at distance greater than $r_{e}$ but within a distance from the sensor. This model reflects the behavior of range sensing devices such as infrared and ultrasound sensors. The probabilistic sensor detection model is shown in Figure 1(b). The distances are measured in units of grid points. Figure 1(b) also illustrates the translation of a distance response from a sensor to the confidence level as a probability value about this sensor response. The coverage for the entire grid sensor field is calculated as the fraction of grid points that exceeds the threshold $c_{t h}$.

\subsection{Optimization of Energy Consumption}

After optimization of coverage, all the deployed sensor nodes move to their own positions. Now our goal is to minimize energy usage in a cluster based sensor network topology by finding the optimal cluster head positions. For this purpose, we assume a power consumption model [10] for the radio hardware energy dissipation where the transmitter dissipates energy to run the radio electronics and the power amplifier, and the receiver dissipates energy to run the radio electronics. This is one of the most widely used models in sensor network simulation analysis. For our approach, both the free space (distance ${ }^{2}$ power loss) and the multi-path fading (distance ${ }^{4}$ power loss) channel models were used. Assume that the sensor nodes inside a cluster have short distance dis to cluster head but each cluster head has long distance Dis to the base station. Thus for each sensor node inside a cluster, to transmit an $l$-bit message a distance dis to cluster head, the radio expends

$$
E_{T S}(l, d i s)=l E_{\text {elec }}+l \varepsilon_{f s} d i s^{2}
$$


For cluster head, however, to transmit an $l$-bit message a distance Dis to base station, the radio expends

$$
E_{T H}(l, D i s)=l E_{\text {elec }}+l \varepsilon_{m p} D_{i s}^{4}
$$

In both cases, to receive the message, the radio expends:

$$
E_{R}(l)=l E_{\text {elec }}
$$

The electronics energy, $E_{\text {elec }}$, depends on factors such as the digital coding, modulation, filtering, and spreading of the signal, here we set as $E_{\text {elec }}=50 \mathrm{~nJ} / \mathrm{bit}$, whereas the amplifier constant, is taken as $\varepsilon_{f_{s}}=10 \mathrm{pJ} / \mathrm{bit} / \mathrm{m}^{2}, \varepsilon_{m p}=0.0013 \mathrm{pJ} / \mathrm{bit} / \mathrm{m}^{2}$.

So the energy loss of a sensor member in a cluster is

$$
E_{s}(l, d i s)=l\left(100+0.01 d i s^{2}\right)
$$

The energy loss of a $\mathrm{CH}$ is

$$
E_{C H}(l, D i s)=l\left(100+1.3 \times 10^{-6} \times D_{i s}{ }^{4}\right)
$$

Since the energy consumption for computation is much less than that for communication, we neglect computation energy consumption here.

Assume $m$ clusters with $n_{j}$ sensor members in the $j^{\text {th }}$ cluster $C_{j}$. The total energy loss $E_{\text {total }}$ is the summation of the energy used by all sensor members and all the $m$ cluster heads:

$$
E_{\text {total }}=l \sum_{j=1}^{m} \sum_{i=1}^{n_{j}}\left(100+0.01 d i s_{i j}^{2}+\frac{100}{n_{j}}+\frac{1.3 \times 10^{-6} D_{i s_{j}^{4}}^{4}}{n_{j}}\right)
$$

Because only 2 terms are related to distance, we can just set the fitness function as:

$$
f=\sum_{j=1}^{m} \sum_{i=1}^{n_{j}}\left(0.01 d i s_{i j}^{2}+\frac{1.3 \times 10^{-6} \mathrm{Dis}_{j}^{4}}{n_{j}}\right)
$$

\section{Performance evaluation}

The PSO starts with a "swarm" of sensors randomly generated. As shown in Fig. 3 is a randomly deployed sensor network with coverage value 0.31 calculated using approximate method mentioned in section 3.1. A linear decreasing inertia weight value from 0.95 to 0.4 is used, decided according to [6]. Acceleration coefficients $c_{1}$ and $c_{2}$ both are set to 2 as proposed in [6]. For optimizing coverage, we have used 20 parti- 
cles, which are denoted by all sensor nodes coordinates, for our experiment in a $50 \times 50$ square sensor network, and the maximum number of generations we are running is 500 . The maximum velocity of the particle is set to be 50 . The other parameters of sensor models are set to be $r=5, r_{e}=3, \lambda=0.5, \beta=0.5, c_{t h}=0.7$. The coverage is calculated as a fitness value in each generation.

After optimizing the coverage, all sensors move to their final locations in setup phase. Now the coordinates of potential cluster heads are set as particles in the sensor network. The communication range of each sensor node is 15 units with a fixed remote base station at $(25,80)$. We start with a minimum number of clusters acceptable in the problem space to be 4 . The node, which will become a cluster head, will not have any restriction on the transmission range. The nodes are organized into clusters by the base station. Each particle will have a fitness value, which will be evaluated by the fitness function (10) in each generation. Our purpose is to find the optimal location of cluster heads. Once the position of the cluster head is identified, if there is no node in that position then a potential cluster head nearest to the cluster head location will become a cluster head.

We also optimized the placement of cluster head in the 2-D space using GA. We used a simple GA algorithm with single-point crossover and selection based on a roulette-wheel process. The coordinates of the cluster head are the chromosomes in the population. For our experiment we are using 10 chromosomes in the population. The maximum number of generations allowed is 500 . In each evolution we update the number of nodes included in the clusters. The criterion to find the best solution is that the total fitness value should be minimal.

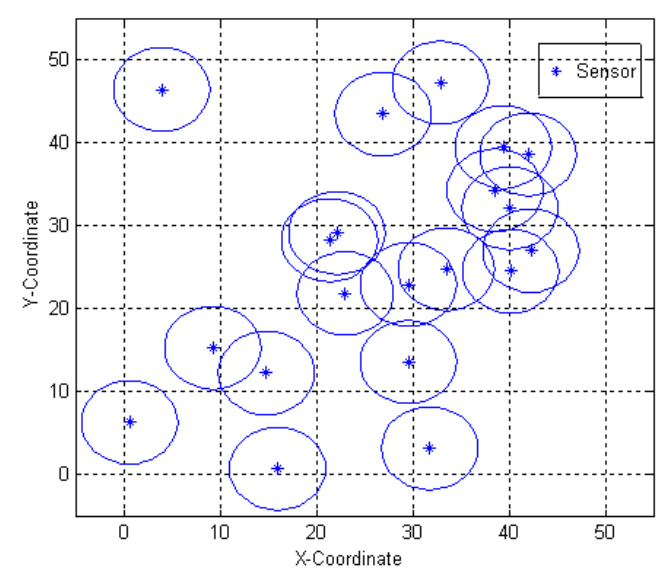

Fig. 3. Randomly deployed sensor network with $r=5$ (Coverage value=0.31) 


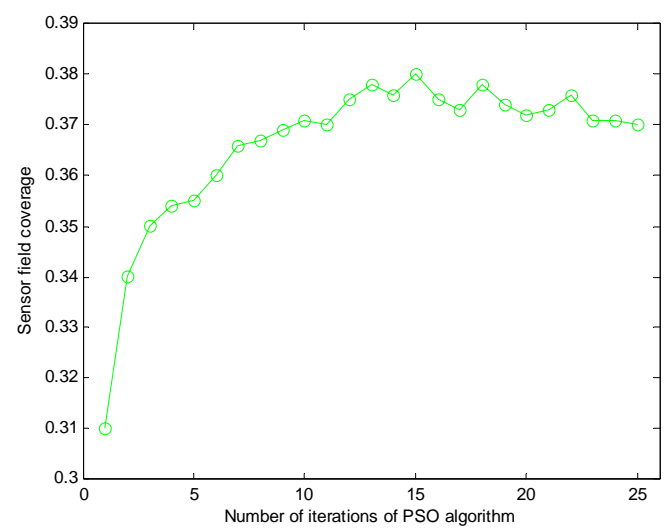

Fig. 4. Optimal coverage achieved using PSO algorithm (probabilistic sensor detection model)

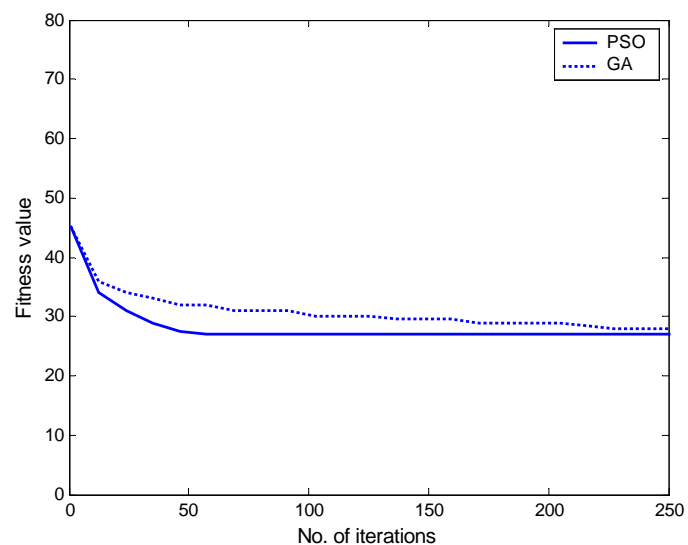

Fig. 5. Comparison of convergence rate between PSO and GA based on Eq. (10)

Fig. 4 shows the improvement of coverage during the execution of the PSO algorithm. Note that the upper bound for the coverage for the probabilistic sensor detection model (roughly 0.38) is lower than the upper bound for the case of binary sensor detection model (roughly 0.628). This due to the fact that the coverage for the binary sensor detection model is the fraction of the sensor field covered by the circles. For the probabilistic sensor detection model, even though there are a large number of grid points that are covered, the overall number of grid points with coverage probability greater than the required level is fewer.

Fig. 5 shows the convergence rate of PSO and GA. We ran the algorithm for both approaches several times and in every run PSO converges faster than GA, which was used in [1] for coverage and lifetime optimization. The main reason for the fast convergence of PSO is due to the velocity factor of the particle. 
Fig. 6 shows the final cluster topology in the sensor network space after coverage and energy consumption optimization when the number of clusters in the sensor space is 4 . We can see from the figure that nodes are uniformly distributed among the clusters compared with the random deployment as shown in Fig 3. The four red stars denote cluster heads, the blue diamonds are sensor members, and the dashed circles are communication range of sensor nodes. The energy saved is the difference between the initial fitness value and the final minimized fitness value. In this experiment, it is approximately 16 .

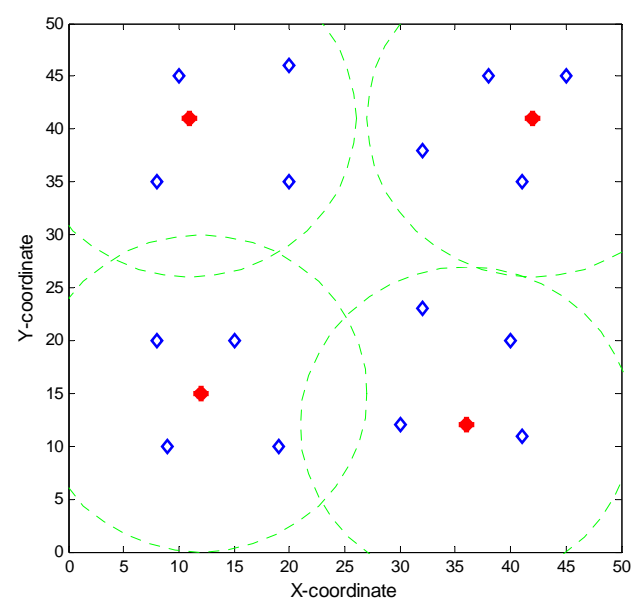

Fig. 6. Energy efficient cluster formation using PSO

\section{Conclusions and Future Work}

The application of PSO algorithm to optimize the coverage in mobile sensor network deployment and energy consumption in cluster-based topology is discussed. We have used coverage as the first optimization objective to place the sensors uniformly based on a realistic probabilistic sensor model, and energy consumption as the second objective to find the optimal cluster head positions. The simulation results show that PSO algorithm has faster convergence rate than GA based layout optimization method while demonstrating good performance.

In the future work, we will take sensor movement energy consumption into account. Moreover, other objectives, such as time and distance for sensor moving will be further studied. 


\section{Acknowledgement}

This research was supported by the Kyung Hee University Research Fund in 2005 (KHU-20050370).

\section{References}

1. Damien B. Jourdan, Olivier L. de Weck: Layout optimization for a wireless sensor network using a multi-objective genetic algorithm. IEEE 59th Vehicular Technology Conference (VTC 2004-Spring), Vol.5 (2004) 2466-2470

2. K. Chakrabarty, S. S. Iyengar, H. Qi and E. Cho: Grid coverage for surveillance and target location in distributed sensor networks. IEEE transactions on computers, Vol.51 (2002) 1448-1453

3. A. Howard, M.J. Mataric and G. S. Sukhatme: Mobile sensor network deployment using potential fields: a distributed, scalable solution to the area coverage problem. Proc. Int. Conf. on distributed Autonomous Robotic Systems (2002) 299-308

4. Y. Zou and K. Chakrabarty: Sensor deployment and target localization based on virtual forces. Proc. IEEE Infocom Conference, Vol. 2 (2003) 1293-1303

5. Kennedy and R. C. Eberhart: Particle Swarm Optimization. Proceedings of IEEE International Conference on Neural Networks, Perth, Australia (1995) 1942-1948

6. Yuhui Shi, Russell C. Eberhart: Empirical study of Particle Swarm Optimization. Proceedings of the 1999 Congress on Evolutionary Computation, Vol. 3 (1999) 1948-1950

7. K.E. Parsopoulos, M.N. Vrahatis: Particle Swarm Optimization Method in Multiobjective Problems. Proceedings of the 2002 ACM symposium on applied computing, Madrid, Spain (2002) 603- 607

8. http://www.swarmintelligence.org/tutorials.php

9. Nojeong Heo and Pramod K. Varshney: Energy-Efficient Deployment of Intelligent Mobile Sensor Networks. IEEE Transactions on Systems, Man, and Cybernetics-Part A: Systems And Humans, Vol. 35, No. 1 (2005) 78 - 92

10. Wendi B. Heinzelman, Anantha P. Chandrakasan, and Hari Balakrishnan: An ApplicationSpecific Protocol Architecture for Wireless Microsensor Networks. IEEE Transactions on Wireless Communications, Vol. 1, No. 4 (2002) 660 - 670

11. A. Elfes: Sonar-based real-world mapping and navigation. IEEE Journal of Robotics and Automation, Vol. RA-3, No. 3 (1987) 249-265

12. Archana Sekhar, B. S. Manoj and C. Siva Ram Murthy: Dynamic Coverage Maintenance Algorithms for Sensor Networks with Limited Mobility. Proc. PerCom (2005) 51-60 